\title{
Hydrophobic and hydrophilic polyrotaxane based movable cross-linkers for thermo-sensitive poly $(N$-isopropylacrylamide $)$ gels
}

\author{
Abu Bin Imran ${ }^{1}$, Takahiro Seki ${ }^{1}$, Kohzo Ito ${ }^{2}$ and Yukikazu Takeoka ${ }^{1}$ \\ ${ }^{1}$ Department of Molecular Design and Engineering, Graduate School of Engineering, Nagoya University, \\ Furo-cho, Chikusa-ku, Nagoya, 464-8603, Japan \\ ${ }^{2}$ Department of Advanced Materials Science, Graduate School of Frontier Sciences, The University of Tokyo, \\ 5-1-5 Kashiwanoha, Kashiwa, Chiba 277-8561, Japan \\ Fax: 81-052-789-4669, e-mail: ytakeoka@apchem.nagoya-u.ac.jp
}

\begin{abstract}
A novel hydrogel has been fabricated using vinyl modified polyrotaxane as a cross-linker. A sparsely dispersed propylene oxide modified $\alpha$-cyclodextrins which were threaded into the long polyethylene glycol $(\mathrm{Mw}=35000)$ and were trapped by bulky 1 -adamantanamine molecules was used as a hydrophilic polyrotaxane, HPR. HPR was modified by a small amount 2-acryloyloxyethylisocyanate monomer to obtain a water soluble polyrotaxane-based movable cross-linker, MHPR. Polymer gels, prepared by free radical polymerization of thermo-sensitive monomer $N$-isopropylacrylamide (NIPA) in presence of MHPR cross-linker, gave transparent, soft and flexible, mechanically strong and fast thermo-sensitive gels. The gel changes its volume isotropically and reaches rapidly to the equilibrium shrunken state after a temperature jump. The hydrophilicity of the cross-linker retains the homogeneity in the gel network and restricts the formation of aggregated globules, which permits the poly(NIPA) chains along with macrocycles to move or rotate freely inside the gel networks under deformation. The movability of the cross-links can strongly minimize their localized stress during deformation. The fascinating characteristics of the gel was compared and contrasted with the gels prepared using using hydrophobic polyrotaxane-based multifunctional and bi-functional
\end{abstract} $N, N$ '-methylene-bis-acrylamide cross-linkers.

Keywords: Hydrogel, polyrotaxane, thermo-sensitivity, morphology, moduli

\section{INTRODUCTION}

Poly( $N$-isopropylacrylamide) hydrogels, due to their fascinating characteristics, inter alia, volume change at ambient temperature, change in opacity, and variation in the gel surface from hydrophilic to a hydrophobic one, and adsorption and desorption of solutes attributable to the phase transition of poly(NIPA) chains at the lower critical solution temperature (LCST), have received a surge of interest for manifold applications. ${ }^{1-6}$ But the inherent weak mechanical properties and critical slowing down during the process of shrinking restrict the use of hydrogels of this variety for widespread use. Research to-date include numerous attempts to obtain mechanically strong and fast shrinking poly(NIPA) gels. The various means used to accelerate shrinking rate of poly(NIPA) gels are the preparation of hydrogels with a bulky heterogeneous structure ${ }^{7}$ or with a macro porous network based on the porosigen technique, ${ }^{8}$ rendering a portion of the gel network hydrophilic ${ }^{9}$ or introduction of freely mobile dangling chains onto the gel network. ${ }^{10}$ Although significant improvements in terms of stimuli sensitivity could be achieved, these approaches are, in most cases, associated with loss of mechanical integrity. This lead to the development of a number of pioneering methods to increase the mechanical strength of polymer gels. ${ }^{11-15}$ Fabrication of sliding gels in which the cross-linking points can slide through the polymer axle has been the most promising one due to their facile syntheses, bio-compatibility and possibilities of modification of hydroxyl group of polyrotaxane $e^{16-18}$ by different functional groups. Gels of this kind are regarded as intermediates between physical and chemical gels where the network is formed by strong covalent interactions among polymer chains like chemical gels and the gels reorient its cross-linking points under deformation like physical gels. However, different polymer systems, in most cases, are not suitable to form complicated polyrotaxane like architecture to obtain sliding gels, and introduction of this novel phenomenon into the gel network is rather difficult and progress in this area is still in the rudimentary stage. Preparation of hydrogels exhibiting fast deswelling rate with adequate mechanical integrity, therefore, still remains an elusive goal and a new route is yet to be opened up. Recently, we reported the synthesis of poly(NIPA) gel using small amount of vinyl modified polyrotaxane as the cross-linker. ${ }^{19}$ The 

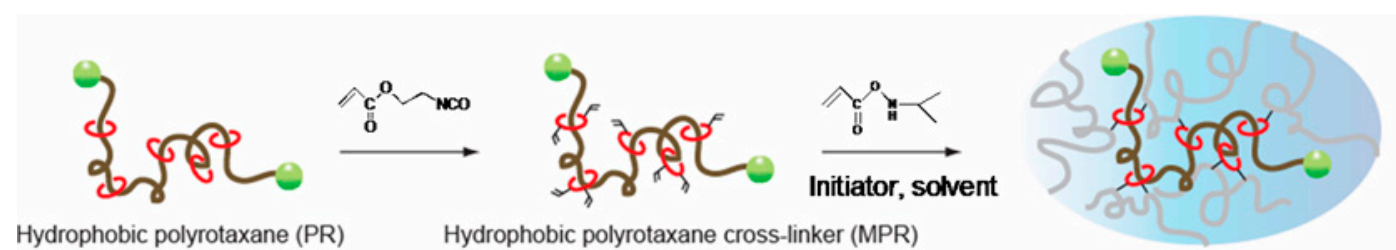

Hydrophobic Rotaxane-NIPA, RwiNx gel
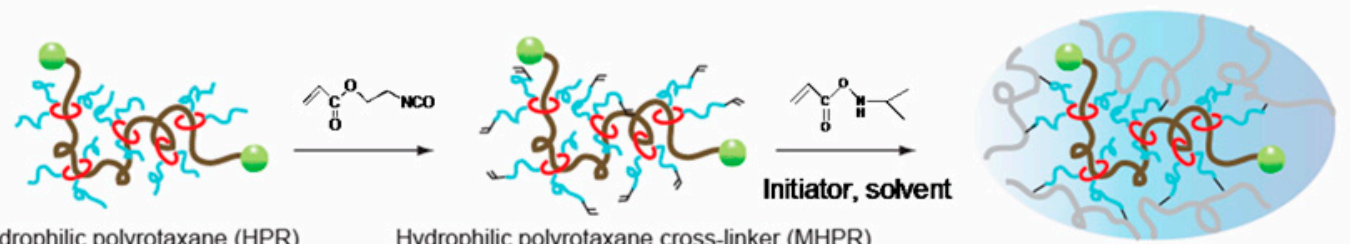

Hydrophilic polyrotaxane (HPR)

Hydrophilic polyrotaxane cross-linker (MHPR)

Hydrophilic Rotaxane-NIPA, RwsNx gel

Scheme 1. Cartoons of the preparation route of hydrophobic and hydrophilic polyrotaxane cross-linkers and their use to form thermo-sensitive polymer gel networks.

prepared gel is very soft and flexible, mechanically strong and exhibits fast deswelling response for a temperature jump in comparison to the typical poly(NIPA) gel (TN gel) but owing to the hydrophobicity of the cross-linker used, the gel has been reported to be macroscopically heterogeneous in aqueous medium.

In this work, we designed a water soluble polyrotaxane cross-linker and fabricated a mechanically improved hydrogel with enhanced optical transparency and thermo-sensitivity. The gel prepared has been characterized and the superiority of the gel has been established over conventional TN gel. The fascinating characteristics of the gel has been compared and contrasted with the gels prepared using hydrophobic polyrotaxane-based movable cross-linkers.

\section{EXPERIMENTAL}

\subsection{Materials}

Polyrotaxanes, PR and HPR, were purchased from Advanced Soft materials Inc., and used without further purifications. $N$-isopropylacrylamide (NIPA) from Kohjin Co. was purified by recrystallization from toluene/n-hexane mixture. 2-Acryloyloxyethyl isocyanate from Showa Denko K.K, $N, N$ '-methylene-bis-acrylamide (BIS) from Acros Organics, $\alpha, \alpha^{\prime}$-azoisobutyronitrile (AIBN) from Kanto Chemical Co., dibutylenetindilaurate (DBTDL) and butyl hydroxyl toluene from Tokyo Kasei Kogyo Co. were used as received without further purifications.

\subsection{Preparation of MPR and MHPR}

PR /HPR ( $1 \mathrm{~g}$ ), DBTDL (catalyst, 2/3 drops), and butylhydroxy toluene (inhibitor, 1.56 $\mathrm{mg})$ were dissolved in dehydrated DMSO $(60 \mathrm{ml})$. 2-acryloyloxyethyl isocyanate $(156 \mathrm{mg})$ dissolved in dehydrated DMSO $(20 \mathrm{ml})$ was then added drop wise to the mixture with continuous stirring in the dark. The reaction was carried out overnight at $40^{\circ} \mathrm{C}$ to ensure complete reaction. MPR/MHPR was collected from the reaction mixtures using excess methanol/acetone followed by keeping at refrigerator. The products were washed several times with methanol/acetone and then freeze dried. The percent yield of polyrotaxane cross-linker MPR or MHPR is approximately $80 \%$.

2.3 Preparation of Rotaxane-NIPA, RN gel and typical poly(NIPA), TN gel

The RN gels were prepared by conventional free radical polymerization in presence of hydrophobic or hydrophilic polyrotaxane cross-linker, MPR or MHPR. Varying amount of NIPA, $2.27 \mathrm{wt} \%$ MPR or MHPR, and $8.1 \mathrm{mM}$ AIBN were dissolved in anhydrous DMSO. The pre-gel solutions were then treated with $\mathrm{N}_{2}$ bubbling to remove any dissolved $\mathrm{O}_{2}$ and were infused to glass slides separated by teflon spacers of varying thickness for the preparation of slab gels. Some micro-capillaries with an inner diameter of 270 $\mu \mathrm{m}$ were inserted there to prepare cylindrical gels. The gelations were performed at $60^{\circ} \mathrm{C}$ for $24 \mathrm{~h}$. The gel samples were washed with DMSO followed by water for two weeks, where solvent was changed at least twice a day. TN gels were prepared following the identical procedure with varying amount of bi-functional cross-linker BIS used as a cross-linker for gelations. For all cases, NIPA was polymerized with almost $100 \%$ conversions, since after gelations no more residue of pre-gel solutions were visualized.

\subsection{Transmittance spectra of gels An optical-fiber}

spectrometer (QE65000, Ocean optics inc.) was used to measure the transmittance spectra of slab gels (thickness $\cong 2 \mathrm{~mm}$ ). Deuterium-halogen 

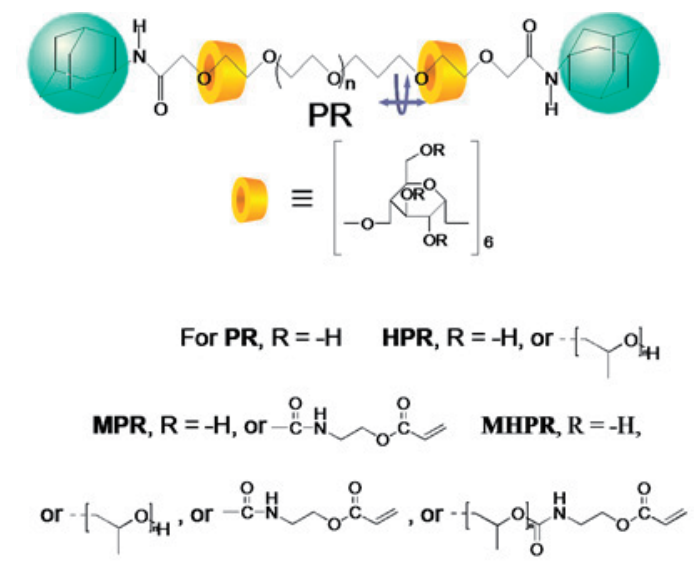

Fig. 1 Chemical structures of water-insoluble polyrotaxane, PR; water-soluble polyrotaxane, HPR; water-insoluble polyrotaxane cross-linker, MPR; and water-soluble polyrotaxane cross-linker, MHPR.

(Mikropack) lamp was selected as a light source.

\subsection{Setup for shrinking kinetics}

The cylindrical gel was immersed in a desired solvent in a glass cell which is connected with two circulators fixed at two different target temperatures. The solvent temperature inside the cell can quickly be switched from one temperature to another only by changing the path of the circulating water. The time to reach at target temperature inside the solvent of the cell was about $70 \pm 5 \mathrm{~s}$. The volume change of the cylindrical gels was monitored under an inverse microscope equipped with color measuring unit. The morphological change of the gels was also studied with simultaneous recording of videos using BUFFALO PCast TV capture software on a computer. Pictures were captured at different time intervals from the recorded video for analysis.

\subsection{Rheometric Solid Analyzer}

The mechanical properties of gels were measured with a Rheometric Solid Analyzer (RSA III of Rheometric Scientific Inc.). The details of the instrumentation and measurement process have been reported earlier. ${ }^{20}$

\section{RESULTS AND DISCUSSION}

Polyrotaxanes used in this study as starting materials for the preparation of hydrophobic and hydrophilic polyrotaxane are of two types: unmodified polyrotaxane, PR and hydroxypropylated polyrotaxane, HPR (Figure 1). The hydrophobic PR consists of $\alpha-C D$ (inclusion ratio $26 \%$ ), poly(ethylene glycol) carboxylic acid $(\mathrm{Mw}=35000)$ and adamantanamine (terminal bulky moieties). The $\mathrm{Mw}$ of $\mathrm{PR}$ is 97040 and polydispersity index, $\mathrm{Mw} / \mathrm{Mn}=1.55$. On the other hand, hydrophilic HPR was achieved by the propylene oxide modification of $\alpha-C D$ of $P R$ whose inclusion ratio is $29 \%$. Molecular substitution (MS) i.e. average mole number of the introduced propylene oxide groups per $\alpha-C D$ unit is 9.18. $(\mathrm{Mw}=125800$ and $\mathrm{Mw} / \mathrm{Mn}=1.4)$. The detail synthetic route of PR and HPR are reported in literature. ${ }^{21} \mathrm{PR}$ is insoluble in most of the solvents especially in water due to the presence of strong inter- and intra-molecular hydrogen bonds among the hydroxyl groups of $\alpha$-CDs. In contrast, introduction of propylene oxide to PR reduces formation of hydrogen bonds among $\alpha$-CDs; consequently the increased dispersity of $\alpha$-CDs onto the PEG chain increases the solubility of HPR in water.

PR and HPR were modified by a 2-acryloyloxyethyl isocyanate having both isocyanate and vinyl groups in the parent structure. The isocyanate end reacts with the hydroxyl groups of PR or HPR to yield polyrotaxane cross-linkers, which we refer to as MPR and MHPR, respectively. The degree of substitution (DS) $(0 \leqq D S \leqq 18)$, i.e., the average number of substituted hydroxyl groups per $\alpha$-CD unit calculated using ${ }^{1} \mathrm{H}$ NMR spectroscopy, has been 2.25 and 1.13 , respectively for MPR and MHPR.

Poly(NIPA) gels were prepared by free radical polymerization of NIPA in the presence of MPR and MHPR cross-linkers in DMSO (Scheme1). For the sake of comparison TN gels were also prepared using bi-functional BIS as a cross-linker. The gel samples used in this study are denoted as $\mathrm{TNx}$ gel, $\mathrm{R}_{\mathrm{wi}} \mathrm{Nx}$ gel, and $\mathrm{R}_{\mathrm{ws}} \mathrm{Nx}$ gels, where TNx gel, $\mathrm{R}_{\mathrm{wi}} \mathrm{Nx}$ gel, and $\mathrm{R}_{\mathrm{ws}} \mathrm{Nx}$ gels stand for typical poly(NIPA) gels, water insoluble rotaxane-NIPA gels and water soluble rotaxane-NIPA gels respectively. The letter $\mathrm{x}$ is an arbitrary constant which represents the amount of NIPA monomer in molar unit (Table 1).

Figure 2 manifests the transmittance spectra of $\mathrm{R}_{\mathrm{wi}} \mathrm{Nx}$ and $\mathrm{R}_{\mathrm{ws}} \mathrm{Nx}$ gels in DMSO and water at room temperature. RN gels are highly transparent in DMSO in the region of visible wavelength, which is indicative of the formation of homogeneous gel networks in nano scale. However, the transparency of $\mathrm{R}_{\mathrm{wi}} \mathrm{Nx}$ gels decreases significantly in water, but $\mathrm{R}_{w s} \mathrm{Nx}$ gels still remain transparent. In the $\mathrm{R}_{\mathrm{wi}} \mathrm{Nx}$ gel, unreacted hydroxyl groups of $\alpha-C D$ of MPR can form intra- and inter-molecular hydrogen bonds with the neighboring MPR; this additional-

Table 1. Sample codes of RNx and TNx gels prepared in DMSO

\begin{tabular}{|c|c|c|c|c|c|}
\hline $\begin{array}{l}\text { Sample codes } \\
\text { of gets }\end{array}$ & $\begin{array}{l}\text { NIPA } \\
\text { (M) }\end{array}$ & $\begin{array}{l}\text { MPR } \\
\text { (wF\%) }\end{array}$ & $\begin{array}{l}\text { MHPR } \\
\text { (WF\%) }\end{array}$ & $\begin{array}{l}\text { Bis } \\
(\mathrm{mM})\end{array}$ & $\begin{array}{l}\text { AIBN } \\
\text { (mM) }\end{array}$ \\
\hline$R_{m} N(1)$ & 1 & 2.27 & $x$ & $x$ & 8.13 \\
\hline$R_{m} N(1)$ & 1 & $x$ & 2.27 & $x$ & 8.13 \\
\hline$R_{m} N(2)$ & 2 & 2.27 & $x$ & $x$ & 8.13 \\
\hline$R_{m} N(2)$ & 2 & $x$ & 2.27 & $x$ & 8.13 \\
\hline $\mathrm{TN}_{\mathrm{a}}(1)$ & 1 & $x$ & $x$ & 16.7 & 8.13 \\
\hline $\mathrm{TN}_{\mathrm{b}}(1)$ & 1 & x & $x$ & 50 & 8.13 \\
\hline $\mathbf{T N}(2)$ & 2 & * & $x$ & 33.4 & 8.13 \\
\hline
\end{tabular}


a)

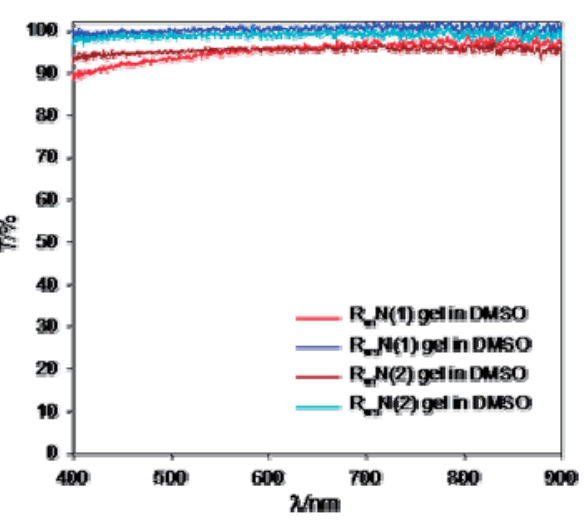

b)

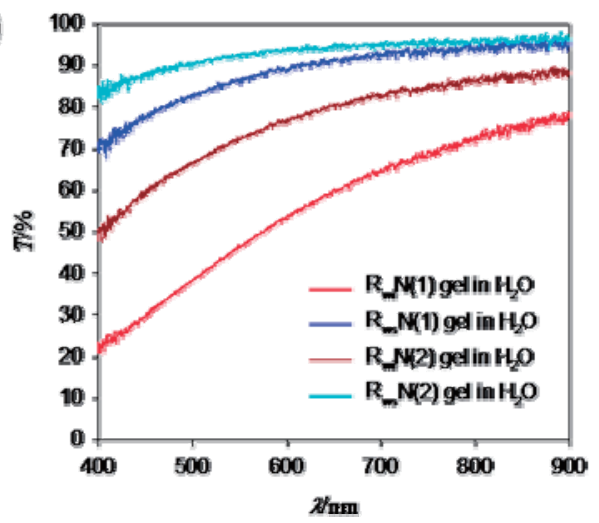

Fig. 2 The changes in the transmittance spectra (visible light wavelength range) of $\mathrm{R}_{\mathrm{wi}} \mathrm{Nx}$ and $\mathrm{R}_{\mathrm{ws}} \mathrm{Nx}$ gels at room temperature $\left(25^{\circ} \mathrm{C}\right)$ a) in DMSO b) in water

a)

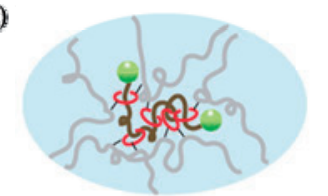

b)

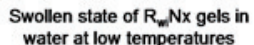
water at low temperatures

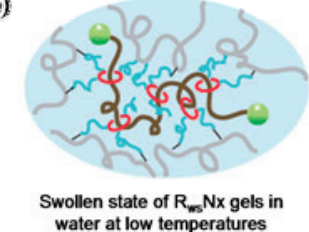

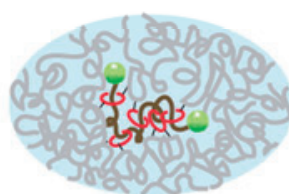

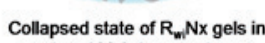
water at high temperatures

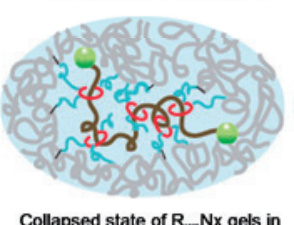

Collapsed state of $\mathrm{R}_{\text {ws }} \mathrm{Nx}$ gels in

Fig. 3 Pictorial representations of swollen and collapsed states of a) $\mathrm{R}_{w i} \mathrm{Nx}$ gels, and b) $\mathrm{R}_{\mathrm{wS}} \mathrm{Nx}$ gels in water at below and above the volume phase transition temperature.

physical interaction as well as water insolubility of the cross-linker MPR, ease the aggregation of cross-linked polymer networks to form clusters in the highly cross-linked region. The sizes of the clusters are larger in size than the wavelength of the visible light to transmit and $\mathrm{R}_{\mathrm{wi}} \mathrm{Nx}$ gels are, therefore, remarkably opaque in water. In contrast, presence of propylene oxide in the MHPR reduces the aggregation of cross-linked polymer chains in the $\mathrm{R}_{\mathrm{ws}} \mathrm{Nx}$ gel networks and the gels remain

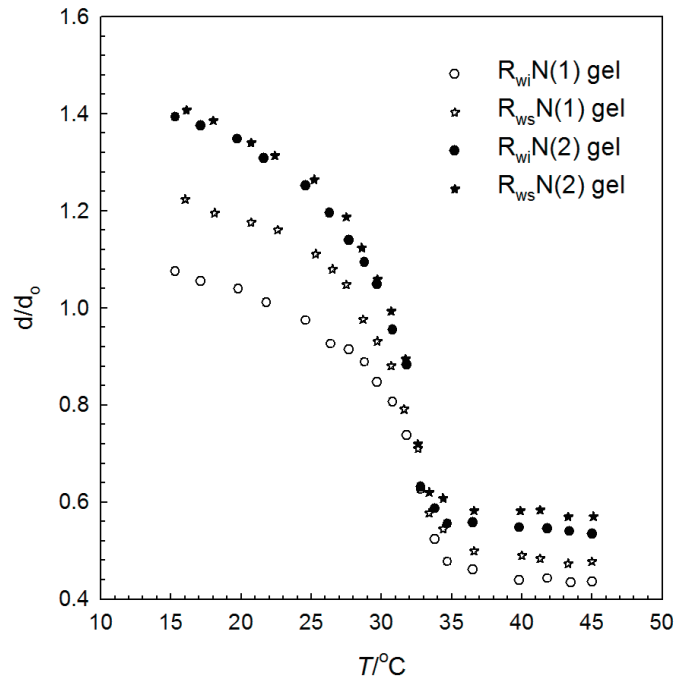

Fig. 4 Equilibrium swelling ratio of cylindrical $\mathrm{R}_{\mathrm{wi}} \mathrm{N}(1), \mathrm{R}_{\mathrm{ws}} \mathrm{N}(1), \mathrm{R}_{\mathrm{wi}} \mathrm{N}(2)$ and $\mathrm{R}_{\mathrm{ws}} \mathrm{N}(2)$ hydrogels in water as a function of temperatures.

transparent in both solvent. The characteristic behaviors of the cross-linkers also imitates in the RN gel networks (Figure 3 )

In Figure 4, the static equilibrium swelling ratio, $\mathrm{d} / \mathrm{d}_{0}$, of $\mathrm{R}_{\mathrm{wi}} \mathrm{Nx}$ and $\mathrm{R}_{\mathrm{ws}} \mathrm{Nx}$ gels, where $\mathrm{d}$ and $\mathrm{d}_{0}$ are the diameter of the equilibrated gels under a certain condition and the diameter of cylindrical gel at a preparative state, respectively, were plotted as a function of temperature. The $\mathrm{R}_{\mathrm{ws}} \mathrm{N}(1)$ gel composed of higher cross-linker density exhibit anomalous swelling behaviors in water compared to hydrophilic moieties incorporated TN gel which leads to an increase in the gel volume and volume phase transition temperature. This irregular behavior resembles the graft or block co-polymer gels ${ }^{22}$ in which the volume phase transition temperature of the gels has been similar to the TN gels but exhibiting higher swelling ratio dependent on the amount of the hydrophilic polymers has been marked. Since relatively much lower cross-linker densities were used to prepare $\mathrm{R}_{\mathrm{wi}} \mathrm{N}(2)$ and $\mathrm{R}_{\mathrm{ws}} \mathrm{N}(2)$ gels, the effect of cross-linker on the swelling ratio are hindered by entanglements of poly(NIPA) chains. The characteristic behaviors of poly(NIPA) chains are in a dominant fashion for the swelling of gels. Hence, irrespective of the nature of the cross-linker, both gels show similar degree of swelling behaviors below the LCST. However, above the LCST, the hydrophilicity of the MHPR inside the $\mathrm{R}_{\mathrm{ws}} \mathrm{N}(2)$ gel network opposes to collapse like $\mathrm{R}_{\mathrm{wi}} \mathrm{N}(2)$ gel.

Figure 5a represents morphological changes of $\mathrm{TN}_{\mathrm{a}}(1), \quad \mathrm{R}_{\mathrm{wi}} \mathrm{N}(1)$ and $\mathrm{R}_{\mathrm{ws}} \mathrm{N}(1)$ hydrogels after a temperature jump from 20 to $40^{\circ} \mathrm{C}$. The $\mathrm{TN}_{\mathrm{a}}(1)$ hydrogel changes its volume through bubble formation and as soon as the volume phase transition temperature is exceeded, bubble formation commences. After a temperature 
a)

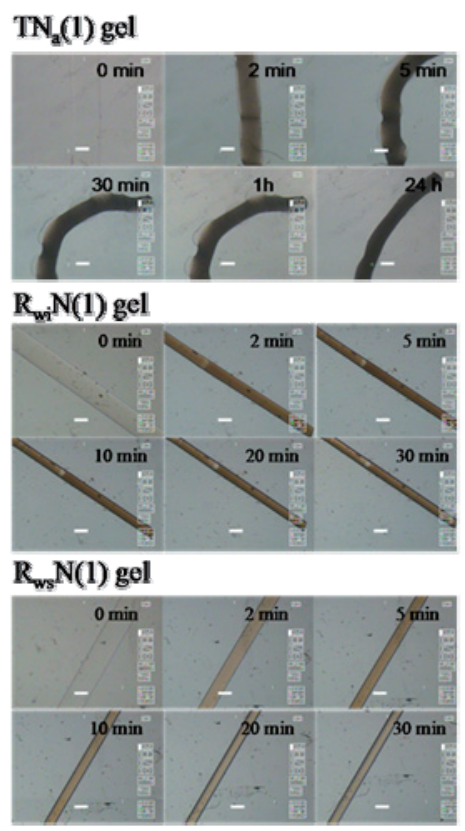

b)

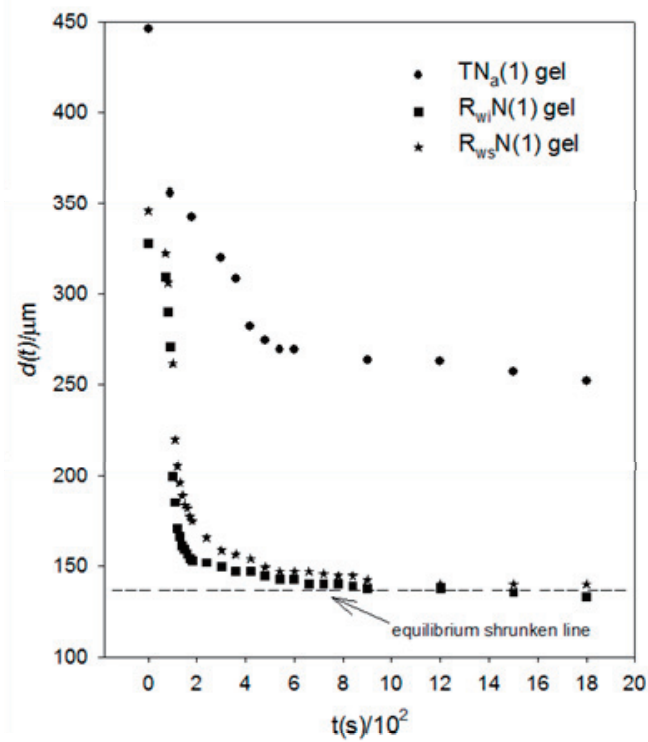

Fig. 5 a) Optical micrographs of morphological changes of sub-micrometer sized cylindrical $\mathrm{TN}_{\mathrm{a}}(1), \mathrm{R}_{\mathrm{wi}} \mathrm{N}(1)$ and $\mathrm{R}_{\mathrm{ws}} \mathrm{N}(1)$ hydrogels after a temperature jump from 20 to $40^{\circ} \mathrm{C}$ b) Fast deswelling kinetics of cylindrical $\mathrm{TN}_{\mathrm{a}}(1), \mathrm{R}_{\mathrm{wi}} \mathrm{N}(1)$, and $\mathrm{R}_{\mathrm{ws}} \mathrm{N}(1)$ hydrogels. $\mathrm{RN}(1)$ gels reach to an equilibrium shrunken state very quickly while $\mathrm{TN}_{\mathrm{a}}(1)$ gel is far from the equilibrium shrunken state.

jump, the shrinking of the gel start at the interface by the formation of micro-phase separated structures which form an impermeable layer on the surface to entrap the water molecule of the bulk gel. When the hydrostatic pressure is stronger enough, the solvent water molecules inside the bulk gel can be expelled out by the disruption of impermeable layer which creates bubble on the surface of the gels. On the other hand, $\mathrm{R}_{\mathrm{wi}} \mathrm{N}(1)$ and $\mathrm{R}_{\mathrm{ws}} \mathrm{N}(1)$ gels exhibit homothetic deformation during shrinking. When the temperature of gels increases above the volume phase transition, both of the gels shrink isotropically without any bubble formation and the deformation rates are very fast. The $\mathrm{R}_{\mathrm{wi}} \mathrm{N}(1)$ gels, after a temperature jump, reaches very close to an equilibrium shrunken state but it retains its opacity for prolonged aging indicating the heterogeneity of gel; while the $\mathrm{R}_{\mathrm{ws}} \mathrm{N}(1)$ gel reaches to an equilibrium transparent shrunken state very rapidly. Faster shrinking kinetics can be observed for homogeneous gels only by changing the cross-linker type. This contrasts to the generalized behavior that homogeneous gel shrinking kinetics is slower than that for an inhomogeneous gel. ${ }^{23}$ Bubble formation is the indication of spatial inhomogeneities in the gel, therefore, it may be speculated that the use of movable cross-linker facilitates complete removal of spatial inhomogeneities.

Figure $5 \mathrm{~b}$ represents the deswlling rate of $\mathrm{TN}_{\mathrm{a}}(1), \mathrm{R}_{\mathrm{wi}} \mathrm{N}(1)$, and $\mathrm{R}_{\mathrm{ws}} \mathrm{N}(1)$ gels after a temperature jump from 20 to $40^{\circ} \mathrm{C}$. The relaxation time, $\tau$ was estimated from the plots of $\ln [d(\mathrm{t}) / d(0)]$ versus $\mathrm{t}$, assuming that the deswelling rate follows first-order kinetics. Two stages relaxation are apparent for $\mathrm{RN}$ gels during shrinking process but most of the volume changes occur in the first stages. The first stage relaxation time, $\tau_{1}$ for $\mathrm{R}_{\mathrm{wi}} \mathrm{N}(1)$ and $\mathrm{R}_{\mathrm{ws}} \mathrm{N}(1)$ gels are $1.26 \times$ $10^{2} \mathrm{~s}$ and $2.11 \times 10^{2} \mathrm{~s}$, respectively, while last stage relaxation time, $\tau_{2}$ are $1.27 \times 10^{4} \mathrm{~s}$ and 6.1 $\times 10^{3} \mathrm{~s}$, respectively. The second stage relaxation for RN gels might come from the final rearrangement of aggregated nuclei to reach equilibrium shrunken state. Hydrophilic cross-linkers in the $\mathrm{R}_{\mathrm{ws}} \mathrm{N}(1)$ gel enhances this rearrangement speed whereas hydrophobic cross-linkers in the $\mathrm{R}_{\mathrm{wi}} \mathrm{N}(1)$ gel causes suppressions. Therefore, the $\mathrm{R}_{\mathrm{wi}} \mathrm{N}(1)$ gel, after a temperature jump, reaches very close to an equilibrium shrunken state after which further volume change is very slow but it retains its opacity for long time aging indicating the heterogeneity of gel; while the $\mathrm{R}_{\mathrm{ws}} \mathrm{N}(1)$ gel reaches to an equilibrium transparent shrunken state very rapidly. This indicates that in the shrunken state, $\mathrm{R}_{\mathrm{ws}} \mathrm{N}(1)$ gel is homogeneous and the gel must not be in the glassy state at the temperature for enhanced movability or flexibility of the cross-links even at higher temperatures. The RN gels networks contain not only cross-linked polymer chains, but also huge dangling free poly(NIPA) chains attached to the active sides of $\alpha-C D$ of polyrotaxane cross-linkers. These dangling poly(NIPA) chains have less mechanical confinements and behave almost similar to the linear free polymer chains. 


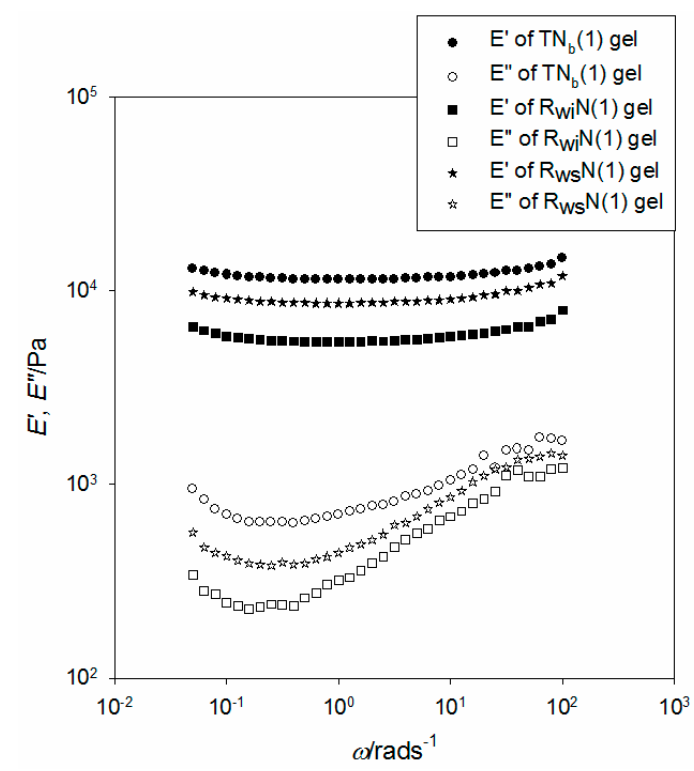

Fig. 6 Strain-controlled dynamic frequency sweep test of storage moduli, $E^{\prime}$ and loss moduli, $E$ ', for $\mathrm{TN}_{\mathrm{b}}(1), \mathrm{R}_{\mathrm{wi}} \mathrm{N}(1)$, and $\mathrm{R}_{\mathrm{ws}} \mathrm{N}(1)$ gels at room temperature (as prepared state).

Cross-linked poly(NIPA) chains in the $\mathrm{R}_{\mathrm{wi}} \mathrm{N}(1)$ and $\mathrm{R}_{\mathrm{ws}} \mathrm{N}(1)$ gels may also exhibit higher degree of freedom due to the presence of movable cross-links. After a temperature jump above the LCST, these free dangling poly(NIPA) chains as well as cross-linked poly(NIPA) chains can quickly collapse to form shrunken phase. Hydrophobic aggregation among shrunken phases then commences to form larger nuclei. At the same time water rich regions combine with each other to form a water channel through which water can be quickly expelled out from the gel networks. The hydrophobic cross-linker MPR used for the synthesis of $\mathrm{R}_{\mathrm{wi}} \mathrm{N}(1)$ gel exists as an aggregated state in water. Hence, the persistent length for sliding of the $\alpha$-CDs along with attached polymer chains decreases to some extent at higher temperatures. As a result, after the first stage major volume collapsing, pinned network structures in the $\mathrm{R}_{\mathrm{wi}} \mathrm{N}(1)$ gel are formed in the second stage to decelerate the deswelling rate. In contrast, the movability of the polymer chains is not altered in the $\mathrm{R}_{\mathrm{ws}} \mathrm{N}(1)$ gel by the aggregation of the cross-linker MHPR. After a temperature jump, the shrinking force developed from the dehydration of poly(NIPA) chains, helps to aggregate shrunken phase quickly owing to the superior movability of the cross-links. The aggregation inside the bulk gel creates huge hydrostatic pressure and water molecules can come out quickly before forming any phase separated pinned network. The shrinking proceeds via the growth of nucleation but do not enter to the unstable spinodal region. The $\mathrm{R}_{\mathrm{ws}} \mathrm{N}(1)$ gel, thereby, immediately turns to an equilibrium shrunken state without any deformation on the gel surfaces.

The $\mathrm{TN}_{\mathrm{b}}(1)$ gel shows relatively faster shrinking speed than the $\mathrm{TN}_{\mathrm{a}}(1)$ gel i.e., when the amount of cross-linker is increased shrinking rate is also enhanced slightly (not shown here) but either gels exhibit very slow deswelling speed than the $\mathrm{RN}(1)$ gels. This is in agreement with literature for the critical slow volume change of conventional poly(NIPA) gels. ${ }^{24}$ The gel is reported to collapse by micro-phase separation because of the spinodal decomposition. The TN gel has lightly and highly cross-linked region; these different types of region give spatial inhomogeneities ${ }^{25}$ to the gel networks. After a temperature jump, the TN gel can not adjust their cross-links region by moving their domains close to each other within a short period of time and consequently gives rise to very slow shrinking rate.

Figure 6 shows strain-controlled dynamic frequency sweep test of storage moduli, $E$ ' and loss moduli, $E$ " for freshly prepared $\mathrm{TN}_{\mathrm{b}}(1), \quad \mathrm{R}_{\mathrm{wi}} \mathrm{N}(1)$, and $\mathrm{R}_{\mathrm{ws}} \mathrm{N}(1)$ gels at room temperature. A frequency controlled $\left(1 \mathrm{rads}^{-1}\right)$ dynamic strain sweep ranging from 0.01 to $20 \%$ was tested first for all gel samples prior to each measurements. RN gels were mechanically very stable in the entire course of measurements, no rupture or cracking was experienced by any of the samples investigated. However, frequent rupture and destruction of the $\mathrm{TNx}$ gel samples at high strain percentage have been apparent for the gels. A $3 \%$ strain which is located in the linear viscoelastic regime for all samples in the strain sweep test was selected for the frequency sweep measurements. The $\mathrm{TN}_{\mathrm{b}}(1)$ gel has higher $E$ ' and E" values compared to the $\mathrm{RN}(1)$ gels in the entire frequencies region which corroborates the stiffness of TN(1) gel netwoks. At low frequencies, the $\mathrm{RN}(1)$ gel gives low $E^{\prime \prime}$ values because of the enhanced movability of the polymer chains along with $\alpha$-CDs at the low frequencies. But at high frequencies polymer chains do not get sufficient time to respond or rearrange the cross-links through the movement of polymer chains for the rapidity of the frequency changes. As a result higher $E$ " values are observed at high frequencies. Poly(NIPA) chains in the TN gel are randomly cross-linked by large number of bi-functional cross-linker BIS in which the cross-links fix the polymer chains to different segments having different lengths. Under deformation all of the stress is, thereby, localized on the shortest poly(NIPA) chains and soon the cross-linked polymer networks start to respond elastically. This endows stiffness of the gels, although they are mechanically fragile in nature. In contrast, $\mathrm{RN}(1)$ gels are inherently soft and flexible in nature since covalently cross-linked PNIPA chains, under deformation, have the ability to slide and rotate through the movable cross-linkers.

\section{CONCLUSION \\ We report here the design of hydrophilic polyrotaxane based movable}


cross-linker, MHPR and employed it for the synthesis of macroscopically homogeneous rotaxane-NIPA hydrogels. The gel exhibits isotropic volume change and very fast deswelling kinetics after a sudden temperature jump. The hydrophilicity of the polyrotaxane cross-linker, MHPR is responsible for the increased deswelling rate. The gel obtained also has a very soft, flexible and substantial gel network similar to the gel prepared by hydrophobic polyrotaxane cross-linker, MPR. The characteristic behaviors of $\mathrm{R}_{\mathrm{ws}} \mathrm{Nx}$ gels have been compared and contrasted with those for $\mathrm{R}_{\mathrm{wi}} \mathrm{Nx}$ and $\mathrm{TNx}$ gels. The softness, mechanically integrity, and fast stimuli sensitivity of the gel can be used potentially for smart sensors, actuators, biomaterials, protein release and drug delivery system.

\section{ACKNOWLEDGEMENT}

Y. T. gratefully acknowledges the financial support of a Grant-in-Aid for Scientific Research from the Ministry of Education, Culture, Sports, Science, and Technology, Japan. NIPA and Karenz AOI were kindly provided by Kohjin Co., Ltd and Showa Denko K. K., respectively.

\section{REFERENCES}

[1] T. Okano, Y. H. Bae, H. Jacobs, and S. W. Kim, J. Controlled Release., 11, 255 (1990).

[2] Y. Kanekiyo, M. Sano, and R. Iguchi, J. Polym. Sci. Part A: Polym. Chem., 38, 1302 (2000).

[3] Y. H. Bae, T. Okano, R. Hsu, and S.W. Kim, Makromol. Chem. Rapid Commun., 8, 481 (1987).

[4] Y. Osada, H. Okuzaki, and H. Hori, Nature, 355, 242 (1992).

[5] S. Bhattacharya, R. A. Moss, H. Ringsdorf, and J. Simon, Langmuir, 13, 1869 (1997).

[6] F. Liu, G. L. Tao, and R. X. Zhuo, Polym. J., 25, 561 (1993).

[7] T. Okajima, I. Harada, K. Nishio, and S. Hirotsu, J. Chem. Phys., 116,9068 (2002).

[8] a. S.X. Cheng, J.T. Zhang, and R.X. Zhuo, J. Biomed. Mater. Res., 67A, 96 (2003). b. J.T. Zhang, S.X. Cheng, and R.X. Zhuo, J. Polym. Sci., Part A: Polym. Chem., 41, 2390 (2003). c. H.R. Oxley, P.H Corkhill, J.H. Fitton, and B.J. Tighe, Biomaterials, 14, 1064 (1993). d. X.Z. Zhang, C.C. Chu, and R.X. Zhuo, J. Polym. Sci., Part A: Polym. Chem., 43, 5490 (2005). e. X.Z. Zhang, and R.X. Zhuo, Eur. Polym. J., 36, 2301 (2000). f. T. Kaneko, T. Asoh, and M. Akashi, Macromol. Chem. Phys., 206, 566 (2005).

[9] a. M. Ebara, T. Aoyagi, K. Sakai, and T. Okano, J. Polym. Sci., Part A: Polym.Chem., 39, 335 (2001). b. A. Gutowska, Y. H. Bae, J. Feijen, and S.W. Kim, J. Controlled Release., 22, 95 (1992).

[10] a. H.K. Ju, S.Y. Kim, and Y. M. Lee, Polymer, 42, 6851 (2001). b. Y. Kaneko, S. Nakamura, K. Sakai, T. Aoyagi, A. Kikuchi, Y. Sakurai, and T. Okano, Macromolecules, 31, 6099 (1998). c. N. Kobuta, T. Matsubara, and Y. Eguchi, J. Appl. Polym. Sci., 70, 1027 (1998). d. K. Sakai, A. Kikuchi, Y. Sakurai, T. Okano, Nature, 374, 240 (1995).

[11] P.G. de Gennes, Physica A., 271, 231 (1999).
[12] Y. Okumura, and K. Ito, Adv. Mater, 13, 485 (2001).

[13] J. P. Gong, Y. Katsuyama, T. Kurokawa, and Y. Osada, Adv. Mater., 15, 1155 (2003)

[14] K. Haraguchi, and T. Takehisa, Adv. Mater., 14, $1120(2002)$

[15] T. Sakai, T. Matsunaga, Y. Yamamoto, C. Ito, R. Yoshida, S. Suzuki, N. Sasaki, M. Shibayama, and U. Chung, Macromolecules, 41, 5379 (2008).

[16] H. L. Frisch, and E. Wasserman, J. Am. Chem. Soc., 83, 3789 (1961).

[17] T. Harrison, and S. Harrison, J. Am. Chem. Soc., 89, 5723 (1967).

[18] C. Zhao, Y. Domon, Y. Okumura, S. Okabe, M. Shibayama, and K. Ito, J. Phys. Condens. Matter., 17, S2841 (2005).

[19] A.B. Imran, Y. Takeoka, T. Seki, T. Kataoka, M. Kidowaki, and K. Ito, Chem. Commun., 41, 5227-5229 (2008).

[20] H. Murayama, A. B. Imran, S. Nagano, T. Seki, M. Kidowaki, K. Ito, and Y. Takeoka, Macromolecules, 41, 1808-1814 (2008).

[21] a. J. Araki, C. Zhao, and K. Ito, Macromolecules, 38, 7524 (2005). b. J. Araki, and K. Ito J. Polym. Sci. Part A: Polym. Chem., 44, 6312 (2006).

[22] G. Chen, A. S. Hoffman, Nature, 373, 49 (1995).

[23] T. Okajima, I. Harada, K. Nishio, and S. Hirotsu, $J$. Chem. Phys., 116, 9068 (2002).

[24] a. S. Hirotsu, Jpn. J. Appl. Phys., Part 2, 37, 284 (1998). b. R. Bansil, G. Liao, and P. Falus, Physica A., 231, 346 (1996). c. Y. Li, G. Wang, and Z. Hu, Macromolecules, 28, 4194 (1995). d. K. Sekimoto, N. Suematsu, and K. Kawasaki, Phys. Rev. A., 39, 4912 (1989). e. A. Onuki, and S. Puri, Phys. Rev. E., 59, R1331 (1999).

[25] a. M. Shibayama, Macromol. Chem. Phys., 199, 1 (1998). b. E. Mendes, R. Oeser, C. Hayes, F. Boue, and J. Bastide, Macromolecules, 29, 5574 (1996). c. H. Hirose, and M. Shibayama, Macromolecules, 31, 5336 (1998).

(Received February 1, 2010; Accepted March 12, 2010) 